\title{
Ethyl Methane Sulphonate (EMS) Induced Mutagenesis in Malaysian Rice (cv. MR219) for Lethal Dose Determination
}

\author{
Ali Benjavad Talebi ${ }^{{ }^{*}}$, Amin Benjavad Talebi ${ }^{2}$, Behzad Shahrokhifar ${ }^{3}$ \\ ${ }^{1}$ Department of Plant Biotechnology, National Institute of Genetic Engineering and Biotechnology (NIGEB), Tehran, Iran; ${ }^{2}$ De- \\ partment of Computer Sciences, Faculty of Science and Information Technology, National University of Malaysia (UKM), Bangi, \\ Malaysia; ${ }^{3}$ Department of Weed Science, Shoushtar Branch, Islamic Azad University, Shoushtar , Iran. \\ Email: "benjavadtalebi.ali@gmail.com
}

Received September $18^{\text {th }}, 2012$; revised October $20^{\text {th }}, 2012$; accepted November $30^{\text {th }}, 2012$

\begin{abstract}
Chemical and physical mutagenesis has been used to increase genetic variability in crop plants. More than 430 new varieties have been derived as mutants of rice (Oryza sativa L.) via the application of different mutagenic agents. Chemical mutagens such as ethyl methane sulphonate (EMS), diepoxybutane-derived (DEB), sodium azide and irradiation (Gamma rays, X-rays and fast neutrons) have been widely used to induce a large number of functional variations in rice and others crops. Among chemical mutagens, the alkylating agent, ethyl methane sulfonate (EMS) is the most commonly used in plants as it causes a high frequency of nucleotide substitutions, as detected in different genomes. In this study, seeds of potential genotype of the popular variety, (Oryza sativa L. spp. Indica cv. MR219) were treated with EMS at concentrations of $0.25 \%, 0.50 \%, 0.75 \%, 1 \%, 1.25 \%, 1.5 \%$ and $2 \%$. Sensitivity to EMS was determined by various measurements on the M1 generation. As concentration of applied EMS increased, will decrease in germination, seedling height, root length and emergence under field conditions was observed in M1 generation as compared to the non-treatment control. Plant height and root length also decreased with increases in EMS mutagenesis in an approximately linear fashion. The LD25 and LD50 values were observed based on growth reduction of seedlings after EMS treatment with $0.25 \%$ and $0.50 \%$ on the rice variety (Oryza sativa L. spp. Indica cv. MR219).
\end{abstract}

Keywords: EMS; Lethal Dose; Chemical Mutagenesis; Ethyl-Methane Sulfonate; Oryza sativa Indica cv. MR219

\section{Introduction}

Chemical mutagens (EMS, DEB and sodium azide) and irradiation (Gamma rays, X-rays and fast neutrons) have been widely used to induce a large number of functional variations in rice. Chemicals induce mainly point mutations, and are thus ideal for producing missense and nonsense mutations, which would provide a series of change-of-function mutations. On the other hand, ionizing radiations normally induce chromosomal rearrangements and deletions [1].

Alkylating agents were the first class of chemical mutagens to be discovered when Auerbach and Robson [2] found the mutagenic effects of mustard gas and related compounds during World War II. Alkylating agents such as mustard gas, methylmethanesulfonate (MMS), ethylmethanesulfonate (EMS), and nitrosoguanidine have several effects on DNA. Because of its potency and ease with which it can be used, EMS is the most commonly

${ }^{*}$ Corresponding author. used chemical mutagen in plants. EMS alkylates guanine bases and leads to mispairing-alkylated $G$ pairs with $T$ instead of $\mathrm{C}$, resulting in primarily $\mathrm{G} / \mathrm{C}-\mathrm{to}-\mathrm{A} / \mathrm{T}$ transitions [1]. EMS mutagenesis in rice involves soaking the seeds in an aqueous solution at a chosen concentration (from $0.2 \%$ to $2.0 \%$ ) for 10 to $20 \mathrm{~h}$ (based on the sensitivity or kill curve of the genotype used). Since EMS produces a large number (genome-wide) of non-lethal point mutations, a relatively small mutant population (approximately 10,000) is sufficient to saturate the genome with mutations. In Arabidopsis, point mutation density can be as high as four mutations per Mb [3-5].

An important advantage of using a common mutagen, such as EMS, is that a substantial body of literature has confirmed its utility in forward genetic screens in a variety of organisms. These include the favorite model animal and model plant for mutagenesis studies in Drosophila melanogaster and Arabidopsis thaliana, respectively. EMS is remarkably consistent, in that apparently similar levels of mutagenesis have been achieved in these 
organisms, despite the approximately 1 billion years of divergence between them. For example, recessive lethal mutations are estimated to occur at similar rates in both cases, with EMS doses causing acceptable levels of sterility and lethality [1]. In addition, direct estimates confirm that base substitution rates are comparable for Arabidopsis seeds soaked in EMS [6,7] and EMS-fed Drosophila males [8], and approximately similar rates were found in a reverse-genetic screen of zebrafish progeny exposed to N-ethyl-N-nitrosourea (ENU) [9]. Thus, chemical mutagenesis causes a high frequency of nucleotide substitutions in a variety of organisms.

Genome size does not appear to be an important factor in EMS mutagenesis because estimates of per gene mutational density found for Arabidopsis appear to be similar for maize [10], which has a 20-fold larger genome size. Therefore, EMS may likely be the mutagen of choice for TILLING (Target Induce Local Lesion in Genome) in plants. However, the toxicity of EMS may vary depending on the species, and other mutagens or post-treatments with antitoxicants may be worth considering [4].

Over the last few years, several new projects have been done with the aim of producing EMS-induced rice mutant populations in the research institutes [1]. Initially LD50 dose is determined, which is used as an optimal dose for mutation induction. By ignoring this step, mutagen dose can either be high or low resulting mutation frequency [11]. The dose assessment for chemicals is determined by varying the concentration and duration of treatment, the solvent used [e.g. dimethyl sulfoxide (DMSO)], or the $\mathrm{pH}$ of the solution [11].

Chemical mutagens (EMS, DES, sodium azide) were also used by treating banana shoot tips to produce variants for tolerance to Fusarium wilt [11]. EMS has been successfully used on chrysanthemum, yielding a frequency of $5.2 \%$ mutants. A wide range of variations in petal color (pink-salmon, light-pink, bronze, white, yellow and salmon color) have been recorded [11]. Luan et al. [12] treated sweet potato (Ipomoea batatas L. callus) with EMS and obtained salt tolerant lines. At that time, the result was attributed to differences in the chemical composition of the chromosomes near the centromere, making them more sensitive to chemical mutagens. While it may indeed be the case, other explanations are possible. For example, genes near the centromere are less likely to be involved in recombination and hence mutations in those genes are less likely to be eliminated through selection. Mutants need at least two generations of meiosis involving chromosome segregation and recombination [13].

Lethal dose, the percentage of test organisms that killed by a specific dosage (of chemicals or radiation), half will die at $\mathrm{LD}_{50}$ [14].

\section{Materials and Methods}

\subsection{Plant Materials}

In this research, 400 seeds of cultivar MR219 (Oryza sativa L. spp. Indica cv. MR219) were chosen for EMSinduced mutagenesis.

\subsection{EMS Mutagenesis}

Seeds of MR219 were placed in a $500 \mathrm{ml}$ flask and ultrapure water was added to about $5 \mathrm{~cm}$ level above the seeds $(\sim 100 \mathrm{ml})$. Seeds were soaked overnight at room temperature for 20 hours. Subsequently, the water was decanted and $50 \mathrm{ml}$ of $0.25 \%, 0.50 \%, 0.75 \%, 1 \%, 1.25 \%$, $1.5 \%$ and $2 \%$ concentrations of EMS ( $/ / v)$ in water was added. Seeds were incubated for 12 hours at room temperature followed by decantation of the EMS and rinsing with $100 \mathrm{ml}$ of ultrapure water (5 times, 4 minutes each) and $200 \mathrm{ml}$ of ultrapure water (4 times, 15 minutes each). Seeds were then rinsed under running tap water for 4 hours before planting in Petri dishes (Table 1).

\subsection{Lethal Dose Study in EMS Mutagenesis}

Based on the EMS-induced mutagenesis, forty seeds were sown for each treatment besides untreated control on filter paper soaked in $5 \mathrm{ml}$ of distillate water in petri dishes. Petri dishes were then placed in an incubator for 7 days at $25^{\circ} \mathrm{C}$. After seven days, the number of seeds that germinated under these conditions was recorded. The grown seeds from each EMS concentration applied and those of the non-treatment control were transferred and planted in rice field soil prepared in plastic pots. Also, the plants were watered with distillate water (just used in research) in the green house. The seedling height and root length of the plants were measured after two weeks (Table 1).

\subsection{Statistical Analysis}

Lethal Dose experiment was organized based on a completely randomized block design with four replications and the random block included seven levels of EMS concentration. Least significant difference (LSD) test at P-values less than 0.01 was used to investigate the Differences in observed averages of all tested parameters between treatment and non-treatment plants. Statistical analysis was conducted using S.A.S software.

\section{Results}

\subsection{Effect of EMS-Induced Mutagenesis on Germination}

Data analysis on number of seed that germinated showed an attendant decrease in germination in M1 generation 
Table 1. EMS mutagenesis scheme for rice.

\begin{tabular}{|c|c|c|c|}
\hline 400 seeds & \multicolumn{2}{|c|}{ Soaking in the $500 \mathrm{ml}$ ultrapure water } & Over night \\
\hline \multicolumn{4}{|c|}{ Seeds classified in batches of 50 seeds/treatment and placed in the flasks } \\
\hline $0.25 \%$ & \multirow{8}{*}{\multicolumn{2}{|c|}{ Concentration of EMS (v/v) in water }} & \multirow[t]{8}{*}{12 hours } \\
\hline $0.50 \%$ & & & \\
\hline $0.75 \%$ & & & \\
\hline $1 \%$ & & & \\
\hline $1.25 \%$ & & & \\
\hline $1.5 \%$ & & & \\
\hline $2 \%$ & & & \\
\hline 0 & & & \\
\hline \multirow{2}{*}{\multicolumn{2}{|c|}{ Rinsing with ultrapure water }} & $100 \mathrm{ml}$ & Repeat for 5 times $/ 4$ min \\
\hline & & with $200 \mathrm{ml}$ & Repeat 4 times/15 min \\
\hline \multicolumn{3}{|c|}{ Rinsing under running tap water } & 4 hours \\
\hline \multicolumn{2}{|r|}{40 Seeds/Treatment } & Soaked in $5 \mathrm{ml}$ of distillate water & Incubate 7 days at $25^{\circ} \mathrm{C}$ \\
\hline \multicolumn{4}{|c|}{ Germination Test } \\
\hline & Grown seeds/treatment & Planting in pots containing rice foeld soil & Incubate in the green house \\
\hline & Plants & Watering with distillate water & 2 weeks \\
\hline
\end{tabular}

with applied increases in concentration of EMS. According to Figure 1 and Table 2, the results obtained indicate that reduction in seed germination occurred with corresponding increase in EMS concentration $(\mathrm{P}<0.01)$.

\subsection{Effect of EMS Mutagenesis on Root Length and Seedling Height}

Analysis of the average of roots length and seedling height showed that EMS-induced mutagenesis imposed significant impact on the seedling height. According to results obtained (Figure 2 and Table 2), seedling height decreased in proportion with increase in applied EMS concentration $(P<0.01)$. Figure 3 and Table 2 in this research, showed that the root length decreased after increasing the concentration of EMS as compared to nontreatment control $(\mathrm{P}<0.01)$. Maximum reduction in root length was observed after mutagenesis was induced with $0.25 \%$ concentration of EMS in MR219.

The highest length of seedling $33.04 \mathrm{~cm}$ has been observed among the control group. Seedling height reduced during EMS-induced mutagenesis. Based on Table 2, the maximum reduction in seedling height was observed when rice MR219 was treated with concentration of $0.25 \%$. Also, maximum root length has been observed highest among seeds in the control group $(96.6 \mathrm{~mm})$ followed closely by the root length and seedlings height at $0.25 \%$ concentration being the highest among the treatment group. The least root length has been recorded when $0.75 \%$ concentration of EMS concentration has

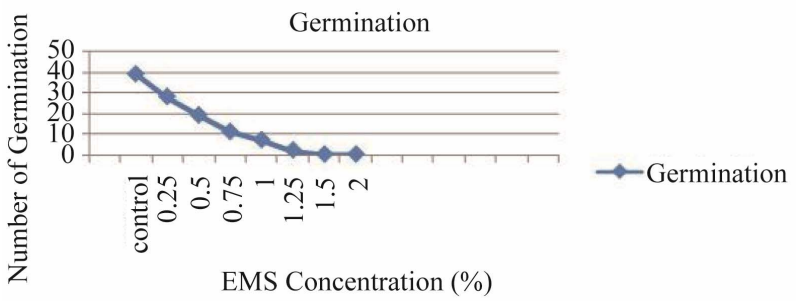

Figure 1. Effect of different concentration of EMS mutagenesis on seed germination.

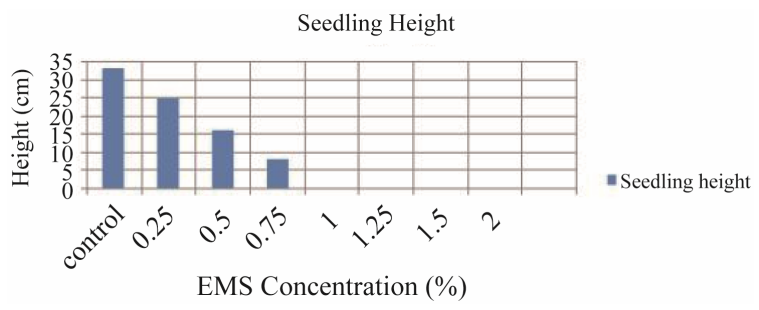

Figure 2. Effect of different concentration of EMS on seedling height.

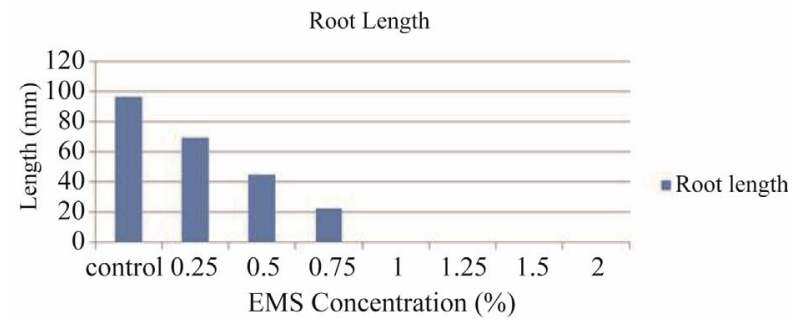

Figure 3. Effect of different concentration on root length. 
Table 2. Mean value of germination, seedling height, root length and emergence following EMS mutagenesis.

\begin{tabular}{|c|c|c|c|c|c|c|}
\hline \multirow{2}{*}{ Treatment } & \multicolumn{2}{|c|}{ Germination } & \multicolumn{2}{|c|}{ Seedling Height (cm) } & \multicolumn{2}{|c|}{ Root Length (mm) } \\
\hline & Actual & $\%$ of control & Actual & $\%$ of control & Actual & $\%$ of control \\
\hline Control & 39 & 100 & $33.04^{*}$ & 100 & $96.6^{*}$ & 100 \\
\hline 0.25 & 28 & 71.79 & $24.58^{*}$ & 74.39 & $69.80^{*}$ & 72.25 \\
\hline 0.50 & 19 & 48.71 & $16.16^{*}$ & 48.91 & $45.15^{*}$ & 46.73 \\
\hline 0.75 & 11 & 28.20 & $8.40^{*}$ & 25.42 & $22.37^{*}$ & 23.15 \\
\hline 1 & 7 & 17.94 & 0 & 0 & 0 & 0 \\
\hline 1.25 & 2 & 5.12 & 0 & 0 & 0 & 0 \\
\hline 1.5 & 0 & 0 & 0 & 0 & 0 & 0 \\
\hline 2 & 0 & 0 & 0 & 0 & 0 & 0 \\
\hline LSD\% & 3.78 & & 5.07 & & 4.44 & \\
\hline C.V\% & 2.13 & & 2.26 & & 2.26 & \\
\hline
\end{tabular}

* Significant at $1 \%$ level, the values are mean of four replicates.

been applied. No readings were observable for seed germination, root length and seedling height when treatment with EMS concentrations above $0.75 \%$ has been applied for the considered genotype.

\section{Discussion}

\subsection{Effect of EMS Mutagenesis on Germination}

It can be observed in the field conditions, EMS mutagenesis caused significant reduction in the germination. This was manifested as significantly decline $(\mathrm{P}<0.01)$ in seed germination as the EMS concentration was increased. Among the chemical mutagens and alkylating agents, EMS has especially been demonstrated to be the most potent.

Previous studies affirm that while the mutagenic response is more or less linear with the dose, polyploids are more tolerant than diploids [13]. According to Figure 1, the current research results showed that after EMS treatment was applied, seed germination was decreased significantly with increasing EMS $(\mathrm{P}<0.01)$.

In concert with a previous study on radiation mutation [15], survival of plants to maturity depends on the nature and extent of chromosomal damage. Increasing frequency of chromosomal harm with increasing radiation dose may be responsible for reduction in germination inability, plant growth and survival. In another study, changes in the germination percentage were attributed to gamma rays treatments [15].

Furthermore, genes near the centromere are more prone to mutagenic treatment than those located farther away. In another study, chlorophyll mutants were frequently observed among EMS treatment group but were rare among those treated with physical mutagens [13]. The stimulating effect of physical mutation on germination may be credited to the activation of RNA or protein synthesis. It may occur during the early stage of germination after the seeds are treated [16].

\subsection{Effect of EMS Mutagenesis on Root Length and Seedling Height}

To identify the biological influences of different physical and chemical mutagens in M1, seedling height is mostly utilized as an index [1]. It has been shown that a linear dependency exists between seedling height and the dosage of physical or chemical mutagens. In concept with this observation, our findings show that decreases in seedling height were because of increases in EMS concentration.

Our results showed that after treatment with EMS was effected in the rice variety MR219, the seedling height significantly $(p<0.01$ ) decreased as compared to the control group. In rice MR219, a significant effect ( $\mathrm{p}<$ 0.01) of the concentration of applied EMS on the root length was observed. Again reduction in root length occurred with each corresponding increase in the concentration of EMS. The symptoms frequently observed in the low-or high-dosage treated plants are enhancement or inhibition of germination, seedling growth, and other biological responses [17].

Regarding the physical mutation study by Wi et al. [17], a hypothesis was presented that low dose irradiation will induce growth stimulation by changing the hormonal signaling network in plant cells or by increasing the anti-oxidative capacity of the cells. Plants can easily overcome daily stress factors such as fluctuations of light intensity and temperature in the growth condition [16] contrast, the high-dose treatment that caused growth inhibition has been ascribed to the cell cycle arrest at G2/M phase during somatic cell division and/or various damages in the entire genome [18]. In this present study, 
variability was measured by mean values of the root length and seedling height both which decreased with increases in the concentration of EMS. According to a physical mutation study by Chaudhuri [19], when radiation is sufficient to reduce the rooting percentages, the root lengths do not exceed a few millimeters in length. Hence due to metabolic disorders in the seeds after radiation treatment, the seeds are unable to germinate [19].

\section{Conclusion}

Lethal Dose was determined by measuring the seed germination, seedling height, root length and emergence of the M1 generation under field conditions. In this experiment, quantitative determinations were applied as a regular procedure. The related data about seedling height, root length and percent of germination were collected and recorded. Variability on observed means was calculated. On the whole, differences between concentration of EMS treatments significantly affected seedling height, root length and germination ( $\mathrm{p}<0.01)$. So, the LD25 and LD50 values observed based on the growth reduction of seedlings after treatments occurred during the application of $0.25 \%$ and $0.50 \%$ concentrations of EMS for the variety MR219.

\section{REFERENCES}

[1] R. Bhat, N. Upadhyaya, A. Chaudhury, C. Raghavan, F. Qiu, H. Wang, J. Wu, K. McNally, H. Leung and B. Till, "Chemical- and Irradiation-Induced Mutants and Tilling," In: N. M. Upadhyaya, Ed., Rice Functional Genomics: Challenges, Progress and Prospects, Springer, New York, 2007, pp. 148-180. doi:10.1007/0-387-48914-2_8

[2] C. Auerbach and J. M. Robson, "Chemical Production of Mutations,” Nature, Vol. 157. No. 3984, 1946, p. 302. doi:10.1038/157302a0

[3] L. Comai and S. Henikoff, "Tilling: Practical SingleNucleotide Mutation Discovery,” The Plant Journal, Vol. 45, No. 4, 2006, pp. 684-694. doi:10.1111/j.1365-313X.2006.02670.x

[4] S. Henikoff and L. Comai, "Single-Nucleotide Mutations for Plant Functional Genomics,” Annual Review of Plant Biology, Vol. 54, No. 1, 2003, pp. 375-401. doi:10.1146/annurev.arplant.54.031902.135009

[5] B. J. Till, S. H. Reynolds, E. A. Greene, C. A. Codomo, L. C. Enns, J. E. Johnson, C. Burtner, A. R. Odden, K. Young and N. E. Taylor, "Large-Scale Discovery of Induced Point Mutations with High-Throughput Tilling," Genome Research, Vol. 13, No. 3, 2003, pp. 524-530. doi:10.1101/gr.977903

[6] C. M. McCallum, L. Comai, E. A. Greene and S. Henikoff, "Targeted Screening for Induced Mutations," Nature Biotechnology, Vol. 18, No. 4, 2000, pp. 455-457. $\underline{\text { doi: } 10.1038 / 74542}$
[7] C. M. McCallum, L. Comai, E. A. Greene and S. Henikoff, "Targeting Induced Locallesions in Genomes (Tilling) for Plant Functional Genomics,” Plant Physiology, Vol. 123, No. 2, 2000, pp. 439-442. doi:10.1104/pp.123.2.439

[8] A. Bentley, B. MacLennan, J. Calvo and C. R. Dearolf, “Targeted Recovery of Mutations in Drosophila," Genetics, Vol. 156, No. 3, 2000, pp. 1169-1173.

[9] E. Wienholds, S. Schulte-Merker, B. Walderich and R. H. A. Plasterk, "Target-Selected Inactivation of the Zebrafish Rag1 Gene,” Science, Vol. 297, No. 5578, 2002, pp. 99-102. doi:10.1126/science.1071762

[10] M. G. Goll and T. H. Bestor, "Histone Modification and Replacement in Chromatin Activation,” Genes \& Development, Vol. 16, No. 14, 2002, pp. 1739-1742. doi:10.1101/gad.1013902

[11] S. M. Jain, "Mutagenesis in Crop Improvement under the Climate Change," Romanian Biotechnological Letters, Vol. 15, No. 2, 2010, p. 89.

[12] Y. S. Luan, J. Zhang, X. R. Gao and L. J. An, "Mutation Induced by Ethylmethanesulphonate (Ems), in Vitro Screening for Salt Tolerance and Plant Regeneration of Sweet Potato (Ipomoea batatas L.)," Plant Cell, Tissue and Organ Culture, Vol. 88, No. 1, 2007, pp. 77-81. doi:10.1007/s11240-006-9183-2

[13] V. L. Chopra, "Mutagenesis: Investigating the Process and Processing the Outcome for Crop Improvement," Current Science, Vol. 89, No. 2, 2005, pp. 353-359.

[14] G. Acquaah, "Principles of Plant Genetics and Breeding," Blackwell Publishing Ltd., Oxford, 2007.

[15] A. L. P. Kiong, A. G. Lai, S. Hussein and A. R. Harun, "Physiological Responses of Orthosiphon Stamineus Plantles to Gamma Irradiation," American-Eurasian Journal of Sustainable Agriculture, Vol. 2, No. 2, 2008, pp. 135-149.

[16] M. S. Abdel-Hady, E. M. Okasha, S. S. A. Soliman and M. Talaat, "Effect of Gamma Radiation and Gibberellic Acid on Germination and Alkaloid Production in Atropa Belladonna L,” Australian Journal of Basic and Applied Sciences. Vol. 2, No. 3, 2008, pp. 401-405.

[17] S. G. Wi, B. Y. Chung, J. S. Kim, J. H. Kim, M. H. Baek, J. W. Lee and Y. S. Kim, "Effects of Gamma Irradiation on Morphological Changes and Biological Responses in Plants,” Micron, Vol. 38, No. 6, 2007, pp. 553-564. doi:10.1016/j.micron.2006.11.002

[18] A. Borzouei, M. Kafi, H. Khazaei, B. Naseriyan and A. Majdabadi, "Effects of Gamma Radiation on Germination and Physiological Aspects of Wheat (Triticum Aestivum L.) Seedlings,” Pakistan Journal of Botany, Vol. 42. No. 4, 2010, pp. 2281-2290.

[19] S. K. Chaudhuri, “A Simple and Reliable Method to Detect Gamma Irradiated Lentil (Lens Culinaris Medik.) Seeds by Germination Efficiency and Seedling Growth Test," Radiation Physics and Chemistry, Vol. 64, No. 2, 2002, pp. 131-136. doi:10.1016/S0969-806X(01)00467-4 